Case Report

\title{
Addison's Disease and Dilated Cardiomyopathy: A Case Report and Review of the Literature
}

\author{
Viktoriya Mozolevska, ${ }^{1}$ Anna Schwartz, ${ }^{1}$ David Cheung, ${ }^{1}$ Bilal Shaikh, ${ }^{1}$ \\ Kapil M. Bhagirath, ${ }^{2}$ and Davinder S. Jassal ${ }^{1,3,4}$ \\ ${ }^{1}$ Institute of Cardiovascular Sciences, St. Boniface General Hospital, University of Manitoba, Winnipeg, MB, Canada \\ ${ }^{2}$ Section of Cardiology, Surrey Memorial Hospital, University of British Columbia, Vancouver, BC, Canada \\ ${ }^{3}$ Section of Cardiology, Department of Internal Medicine, University of Manitoba, Winnipeg, MB, Canada \\ ${ }^{4}$ Department of Radiology, St. Boniface General Hospital, University of Manitoba, Winnipeg, MB, Canada \\ Correspondence should be addressed to Davinder S. Jassal; djassal@sbgh.mb.ca
}

Received 15 September 2016; Accepted 31 October 2016

Academic Editor: Takatoshi Kasai

Copyright (C) 2016 Viktoriya Mozolevska et al. This is an open access article distributed under the Creative Commons Attribution License, which permits unrestricted use, distribution, and reproduction in any medium, provided the original work is properly cited.

Addison's disease is often accompanied by a number of cardiovascular manifestations. We report the case of a 30 -year-old man who presented with a new onset dilated cardiomyopathy due to Addison's disease. The clinical presentation, treatment, and outcomes of this rare hormone mediated cardiac disorder are reviewed.

\section{Introduction}

Addison's disease, also known as primary adrenal insufficiency, is associated with a decreased production of glucocorticoid and mineralocorticoid hormones from the adrenal cortex [1]. Although autoimmune adrenalitis is considered to be the major cause of Addison's disease in up to $90 \%$ of diagnosed individuals, prevalent in female patients between 30 and 50 years of age, other etiologies include infectious, drug induced, and/or genetic factors [2, 3]. Common manifestations of this condition are hyponatremia, hyperkalemia, and/or hypoglycemia along with mucosal and skin hyperpigmentations [2,3]. Although cardiovascular manifestations of Addison's disease include hypotension, syncope, and arrhythmias, the development of a dilated cardiomyopathy and heart failure are an uncommon life-threatening complication [48]. We describe a rare case of a 30-year-old male with a dilated cardiomyopathy in the context of Addison's disease and review all 6 adult cases of this cardiac manifestation published to date [4-8]. We evaluate the clinical presentation, treatment, and outcomes of these cases and discuss the diagnosis, pathophysiology, and treatment of this rare hormone mediated cardiac disorder.

\section{Case Report}

A 30-year-old previously healthy male presented to the emergency department with constitutional symptoms of weakness, fatigue, dizziness, and anorexia. A baseline EKG and chest X-ray were within normal limits. He was diagnosed with primary adrenal insufficiency presenting with biochemical evidence of high ACTH (>278 pmol/L) and low cortisol levels $(32 \mathrm{nmol} / \mathrm{L}$ ) and started on prednisone $5 \mathrm{mg}$ po od and fludrocortisone $0.1 \mathrm{mg}$ po od. Two weeks later, he presented with a 5-day history of orthopnea and increasing dyspnea on minimal exertion. On presentation, the blood pressure was $104 / 74 \mathrm{~mm} \mathrm{Hg}$ with a heart rate of 90 beats per minute. The jugular venous pressure was elevated at the angle of the jaw with displacement of the cardiac apex and normal heart sounds. There were diminished breath sounds bilaterally in both lower lung fields without evidence of peripheral edema. The serum sodium was mildly reduced at $132 \mathrm{mmol} / \mathrm{L}$ (normal range: $135-145 \mathrm{mmol} / \mathrm{L}$ ) while the potassium was increased at $5.6 \mathrm{mmol} / \mathrm{L}$ (normal range: $3.5-5.0 \mathrm{mmol} / \mathrm{L}$ ). There was a markedly elevated $\mathrm{N}$-terminal brain natriuretic peptide of $4515 \mathrm{pg} / \mathrm{mL}$ (normal range $<125 \mathrm{pg} / \mathrm{mL}$ ), consistent with clinical and biochemical evidence of acute heart failure. The serum TSH, urine toxicology, and metanephrines were 


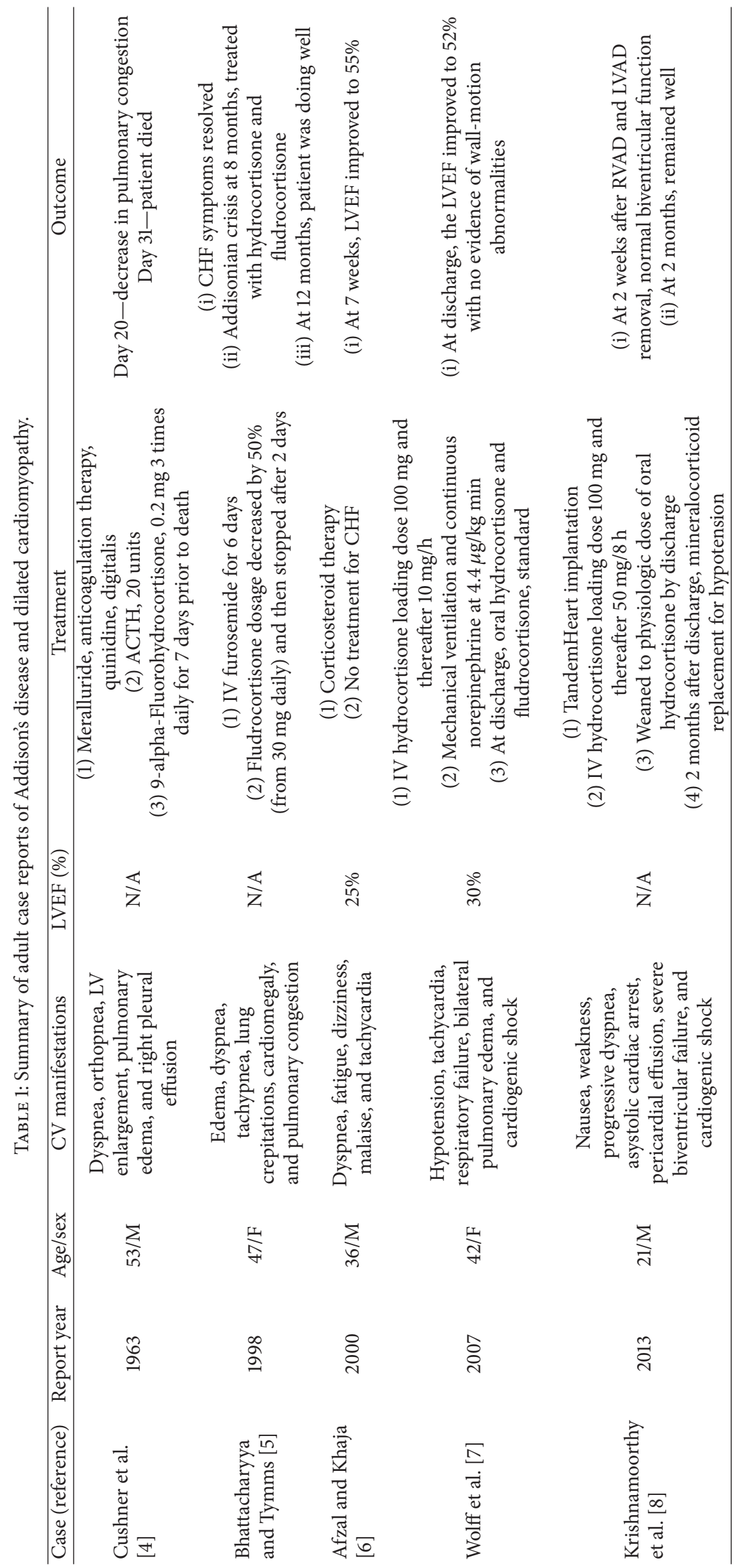




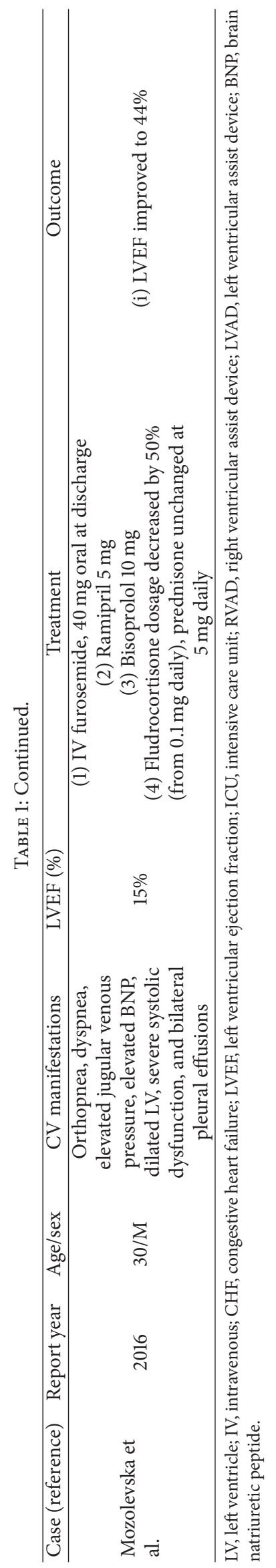


within normal limits. A 12-lead EKG demonstrated poor Rwave progression across the precordial leads with evidence of a left anterior fascicular block. Chest X-ray findings were consistent with bilateral pleural effusions, vascular redistribution, and interstitial edema. Transthoracic echocardiography (TTE) confirmed a dilated left ventricle (LV), severe LV systolic dysfunction with an ejection fraction of $15 \%$, and moderate functional mitral regurgitation. Cardiac magnetic resonance imaging with late gadolinium enhancement was normal with no evidence of a viral myocarditis nor infiltrative cardiomyopathy. Cardiac catheterization demonstrated normal coronaries consistent with the diagnosis of a nonischemic dilated cardiomyopathy. A computed tomographic scan of the abdomen revealed hyperdense adrenal glands with central necrotic areas of hypoattenuation and peripheral enhancement, consistent with the recent diagnosis of primary adrenal insufficiency. With a new diagnosis of acute heart failure due to primary Addison's disease, the patient was admitted for medical treatment with parenteral diuretics, ACE inhibition, beta blockade, and a decrease in the fludrocortisone dosage (that was started 2 weeks before). A 6-month follow-up multigated acquisition scan (MUGA) revealed an improvement in LV ejection fraction to $40-45 \%$.

\section{Discussion}

With an incidence of approximately 120 cases per million in the population, Addison's disease is a rare long term endocrine disorder in which the adrenal glands produce insufficient steroid hormones, including glucocorticoids and mineralocorticoids $[1-3,9]$. The clinical presentation of Addison's disease encompasses a multitude of systemic symptoms including fatigue, weight loss, salt craving, and joint and back discomfort $[1-3,9]$. Individuals with primary adrenal insufficiency may also experience a number of cardiovascular symptoms including hypotension, arrhythmias, and congestive heart failure [4-8]. In fact, the development of a dilated cardiomyopathy due to Addison's disease is extremely rare [4-8]. Although the exact etiology of heart failure due to Addison's disease remains unknown, a number of potential mechanisms include (i) reduced effects of cortical hormones on the LV myocardium; (ii) poor alimentation with low glycogen reserves; (iii) hemoconcentration, low blood volume, and reduced coronary blood flow; and (iv) disturbances in electrolyte levels [9].

After a systematic review of the literature of all adult cases of Addison's disease and dilated cardiomyopathy, a total of 6 published cases (including the current report) were identified as shown in Table 1 [4-8]. The mean age was 38 years (range 21-53) with a male to female ratio of $2: 1$. Common cardiovascular features at presentation were hypotension, dyspnea, tachycardia, and pulmonary edema. Our case was unique in that the patient developed bilateral pleural effusions and the lowest LVEF as measured by TTE. Moreover, our case was only the second in which a patient developed symptoms of acute $\mathrm{CHF}$ following administration of hormone replacement therapy (HRT) and the one with the shortest time lapse between the two events of only 6 days (Table 1) [4-8].
In patients with Addison's disease, dual HRT with glucocorticoids and mineralocorticoids is a necessity $[1-3,10]$. Although oral hydrocortisone and fludrocortisone are the mainstay of treatment in this patient population $[1-3,10]$, the use of these medications may need to be altered in the setting of a concomitant heart failure crisis. In particular, fludrocortisone corrects hypotension by increasing sodium retention, thereby increasing systemic afterload. However, in the presence of a dilated cardiomyopathy and reduced LVEF, increased afterload by fludrocortisone may precipitate acute congestive heart failure [4-8]. In individuals with Addison's disease who experience acute cardiovascular symptoms due to the development of a dilated cardiomyopathy, the dosage of fludrocortisone may need to be decreased or stopped altogether in order to correct the acute heart failure syndrome. In 4 of the 6 cases reviewed in Table 1, the patients were able to tolerate a carefully balanced dose of fludrocortisone after appropriate treatment of the underlying heart failure symptoms with medical therapy including diuretics, ACE inhibition, and beta blockade [4-8]. These agents, while appropriate for acute heart failure in the setting of a reduced LVEF, may interfere with the effectiveness of fludrocortisone in correcting hormonal deficiency, necessitating careful hemodynamic monitoring of the patient's condition throughout the treatment period.

\section{Conclusion}

A multidisciplinary approach must be sought in the management of patients with dilated cardiomyopathy secondary to Addison's disease. HRT dosage should be optimized, heart failure therapy should be initiated, and patients should be closely monitored for future cardiovascular complications.

\section{Competing Interests}

No commercial relationship existed in the form of financial support or personal financial interest in relation to any part of the manuscript.

\section{References}

[1] L. K. Nieman and M. L. Chanco Turner, "Addison's disease," Clinics in Dermatology, vol. 24, no. 4, pp. 276-280, 2006.

[2] S. Bensing, A. Hulting, E. S. Husebye, O. Kämpe, and K. Løvås, "Management of endocrine disease: epidemiology, quality of life and complications of primary adrenal insufficiency: a review," European Journal of Endocrinology, vol. 175, no. 3, pp. R107R116, 2016.

[3] E. Charmandari, N. C. Nicolaides, and G. P. Chrousos, "Adrenal insufficiency," The Lancet, vol. 383, no. 9935, pp. 2152-2167, 2014.

[4] G. B. Cushner, S. F. Zahler, and A. G. Hills, "Untreated Addison's disease complicated by pulmonary congestion due to left ventricular failure," Annals of Internal Medicine, vol. 58, pp. 341-346, 1963.

[5] A. Bhattacharyya and D. J. Tymms, "Heart failure with fludrocortisone in Addison's disease," Journal of the Royal Society of Medicine, vol. 91, no. 8, pp. 433-434, 1998. 
[6] A. Afzal and F. Khaja, "Reversible cardiomyopathy associated with Addison's disease," Canadian Journal of Cardiology, vol. 16, no. 3, pp. 377-379, 2000.

[7] B. Wolff, K. Machill, I. Schulzki, D. Schumacher, and D. Werner, "Acute reversible cardiomyopathy with cardiogenic shock in a patient with Addisonian crisis: a case report," International Journal of Cardiology, vol. 116, no. 2, pp. e71-e73, 2007.

[8] A. Krishnamoorthy, R. J. Mentz, K. A. Hyland et al., "A crisis of the heart: an acute reversible cardiomyopathy bridged to recovery in a patient with addison's disease," ASAIO Journal, vol. 59, no. 6, pp. 668-670, 2013.

[9] I. I. Goodof and C. M. Macbryde, "Heart failure in Addison's disease with myocardial changes of potassium deficiency," The Journal of Clinical Endocrinology \& Metabolism, vol. 4, no. 1, pp. 30-34, 2008.

[10] W. Arlt and B. Allolio, "Adrenal insufficiency," The Lancet, vol. 361, no. 9372, pp. 1881-1893, 2003. 


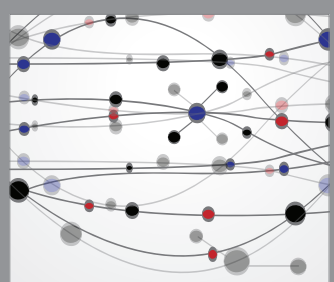

The Scientific World Journal
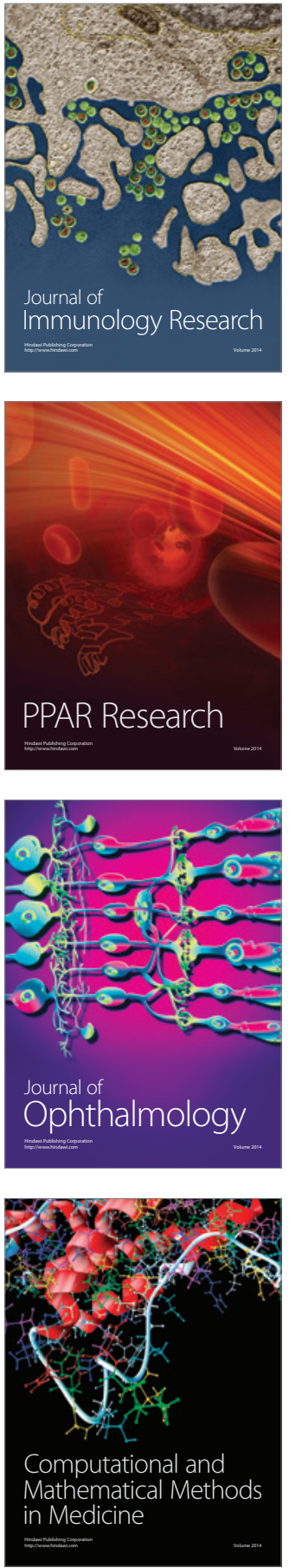

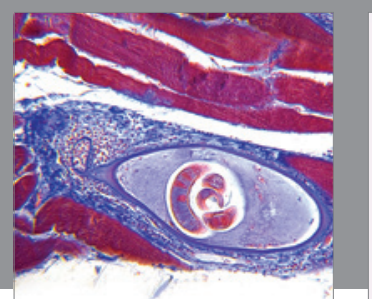

Gastroenterology Research and Practice

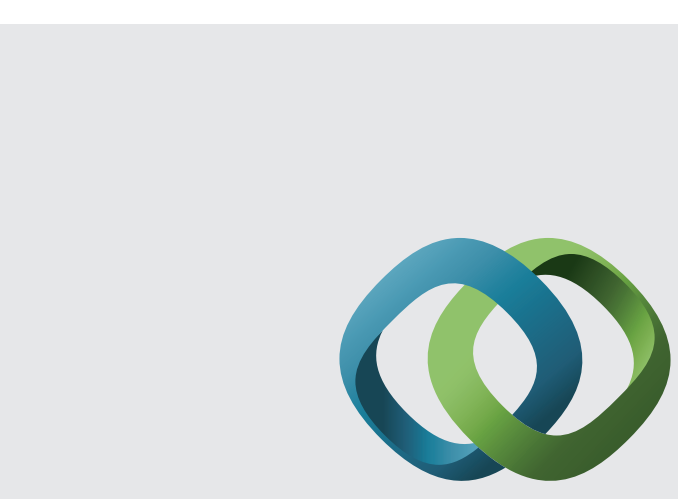

\section{Hindawi}

Submit your manuscripts at

http://www.hindawi.com
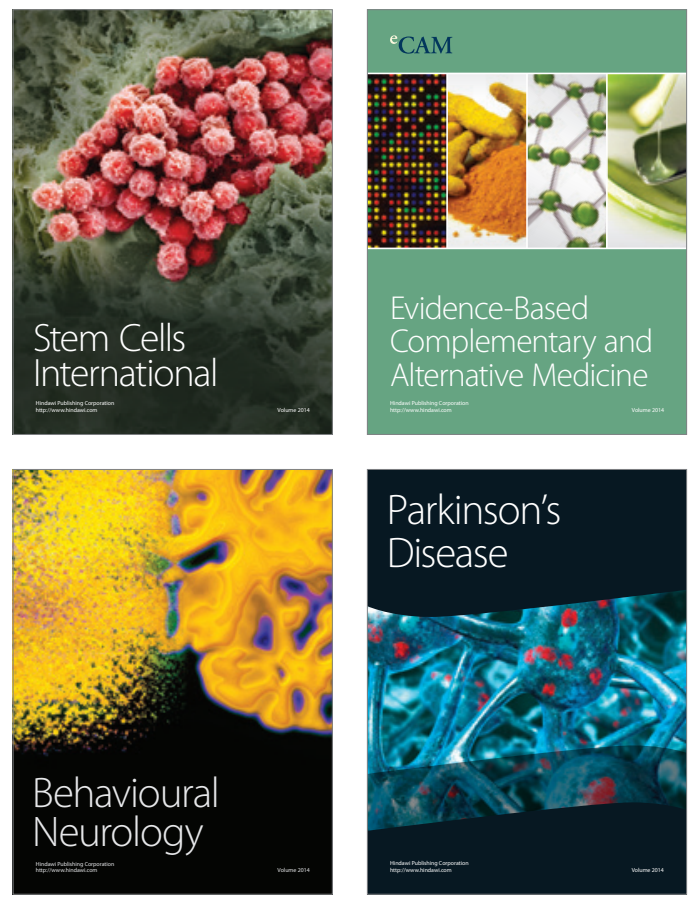
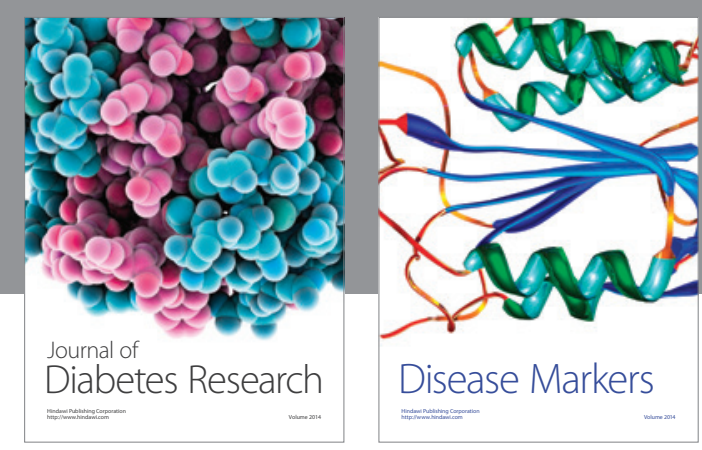

Disease Markers
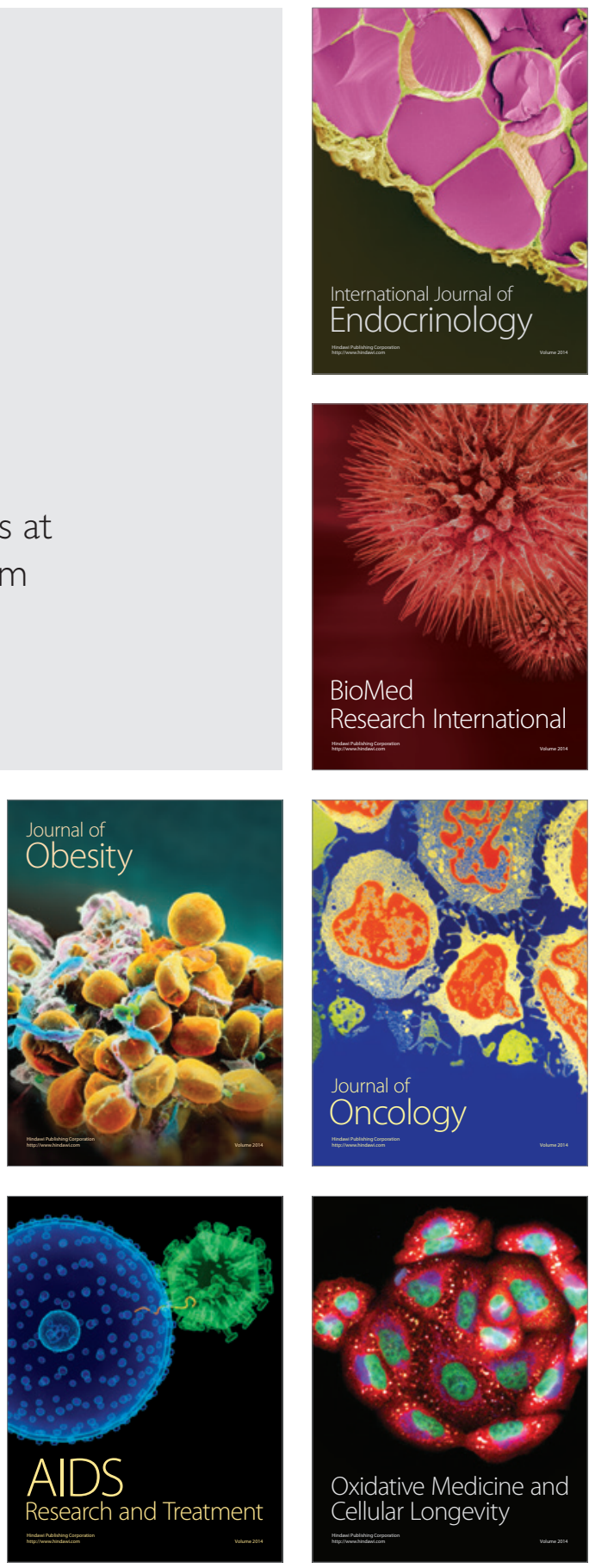